\title{
Estimation of Trabecular Thickness in Gray-Scale Images Through Granulometric Analysis
}

\author{
Rodrigo Moreno $^{a b}$, Magnus Borga ${ }^{a c}$ and Örjan Smedby ${ }^{a b}$ \\ ${ }^{a}$ Center for Medical Image Science and Visualization (CMIV), Linköping University, Sweden \\ ${ }^{b}$ Department of Medical and Health Sciences (IMH), Linköping University, Sweden \\ ${ }^{c}$ Department of Biomedical Engineering (IMT), Linköping University, Sweden
}

\begin{abstract}
This paper extends to gray-scale the method proposed by Hildebrand and Rüegsegger for estimating thickness of trabecular bone, which is the most used in trabecular bone research, where local thickness at a point is defined as the diameter of the maximum inscribed ball that includes that point. The proposed extension takes advantage of the equivalence between this method and the opening function computed for the granulometry generated by the opening operation of mathematical morphology with ball-shaped structuring elements of different diameter. The proposed extension (a) uses gray-scale instead of binary mathematical morphology, (b) uses all values of the pattern spectrum of the granulometry instead of the maximum peak as used for binary images, (c) corrects bias on local thickness estimations generated by partial volume effects, and (d) uses the gray-scale as a weighting function for global thickness estimation. The proposed extension becomes equivalent to the original method when it is applied to binary images. A new non-flat structuring element is also proposed in order to reduce the discretization errors generated by traditional flat structuring elements. Translation invariance can be attained by up-sampling the images through interpolation by a factor of two. Results for synthetic and real images show that the quality of the measurements obtained through the original method strongly depends on the binarization process, whereas the measurements obtained through the proposed extension do not. Consequently, the proposed extension is more appropriate for images with limited resolution where binarization is not trivial.
\end{abstract}

Keywords: Thickness estimation, trabecular bone, mathematical morphology, granulometry

\section{INTRODUCTION}

Estimation of trabecular bone thickness has great relevance in bone research, since changes of this parameter has been associated to osteoporosis ${ }^{1,2}$, osteoarthritis ${ }^{3}$ and changes on the mechanical competence of bone ${ }^{4,5}$.

Although it is not the case for arbitrary shapes, thickness can be unequivocally defined for two specific cases: a) for plate-like objects, as the shortest distance between two parallel faces of the object, and b) for rod-like objects, as the diameter of the object. Thus, thickness of trabecular bone can be estimated under the assumption that it is a network of plate- and rod-like trabeculae.

The most widely used method for estimating thickness on segmented images was proposed by Hildebrand and Rüegsegger $(\mathrm{HR})^{6}$. The method comprises three steps (cf. Figure 1). First, the medial axis and the Euclidean distance transform are computed for the segmented trabecular bone. Second, local thickness is defined for every point $x$ inside the trabecular bone as the diameter of largest inscribed sphere centered at any point in the medial axis that includes $x$. That is, the thickness for the image $f$ at $x, T h(f)(x)$, is estimated as:

$$
T h(f)(x)=2 \max \left\{t \mid x \in B_{t}(c), \forall B_{t}(c) \subseteq X\right\},
$$

where $B_{t}(c)$ is a ball of radius $t$ with its center at a point $c$ which lies in the medial axis of the segmented trabecular bone $X$. Finally, global thickness is estimated as the mean of the local thickness at every point inside the object.

Corresponding address: Linköping University, Campus US, 581 85, Linköping, Sweden, E-mail: \{rodrigo.moreno,magnus.borga,orjan.smedby\}@liu.se 


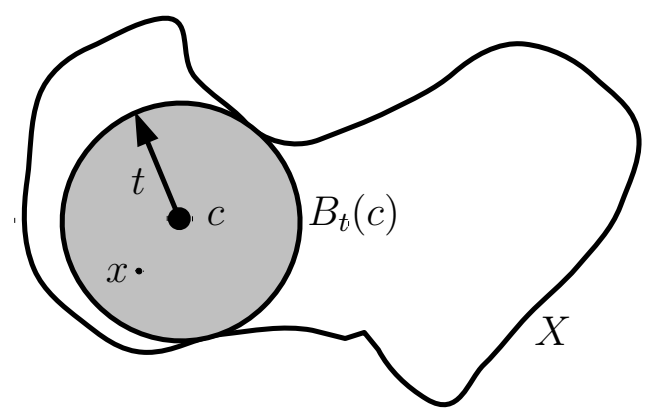

Figure 1. HR computes the thickness at $x$ as twice the radius $t$ of the maximum inscribed sphere $B_{t}(c)$ that includes $x$.

The main drawback of this method is that it has only been defined for binary images. Thus, a previous step of binarization is required, since raw data are usually in gray-scale. For low quality images, this step can lead to loss of relevant information. Unfortunately, this is usually the case for in vivo examinations of trabecular bone.

Two approaches have been followed for estimating thickness in gray-scale images. First, in ${ }^{7}$, the computation of the Euclidean distance transform was replaced by a fuzzy variant ${ }^{8}$ where distances are weighted by the gray-scale values. However, this weighting can result in large underestimations of thickness. More recently, an improvement of this method was proposed $\mathrm{in}^{9}$. However, this method may also introduce large distortions on the Euclidean distance. A second approach applied a Haar-like wavelet followed by a multiscale analysis ${ }^{10}$. However, the negative values present in mother wavelets make difficult the estimation of thickness for objects with non-spherical shapes by applying this strategy. Instead, this paper explores a direct extension of HR using the theory of granulometries of mathematical morphology ${ }^{11}$.

Coeurjolly ${ }^{12}$ showed that the opening function of mathematical morphology computed with ball-shaped structuring elements in binary images is equivalent to the first two steps of HR, that is, the opening function is an equivalent alternative to compute the local thickness at every point of the object. The natural consequence of this result is that HR can be extended to gray-scale images by applying the the same methodology using gray-scale instead of binary mathematical morphology. Thus, the aim of this paper is to propose an accurate method to compute HR for gray-scale images based on the equivalence between this method and the opening function of mathematical morphology.

The paper is organized as follows. Section 2 summarizes some concepts from granulometries and mathematical morphology. Section 3 introduces the proposed formulation for computing thickness in gray-scale. Section 4 deals with discretization issues of granulometries. Section 5 shows some experimental results. Finally, Section 6 discusses the obtained results and makes some final remarks.

\section{CONTINUOUS GRANULOMETRIES}

This section recalls some concepts related with continuous granulometries which are required by the method presented in the next section. The theory of granulometries was originally introduced by Matheron ${ }^{11}$ to compute size distributions of grains in digital images. Intuitively, a granulometry is analog to a sieving process with sieves of different hole sizes, where the size of a grain is determined by the minimum size of the hole that sifts the grain.

More formally, let $f$ and $g$ be continuous functions and $\Psi_{t}(f)$ be a family of functions with a scale parameter $t \geq 0 . \Psi_{t}(f)$ is a granulometry if and only if it complies with the following properties: ${ }^{13}$

$$
\begin{array}{cl}
\text { Anti-extensivity: } & \Psi_{t}(f)(x) \leq f(x) \\
\text { Increasingness: } & \text { if } f(x) \leq g(x), \forall x, \text { then } \\
& \Psi_{t}(f)(x) \leq \Psi_{t}(g)(x), \\
\text { Idempotence: } & \Psi_{t}(f)=\Psi_{t}\left(\Psi_{t}(f)\right) \\
\text { Absorption: } & \Psi_{t_{1}}\left(\Psi_{t_{2}}(f)\right)=\Psi_{t_{2}}\left(\Psi_{t_{1}}(f)\right) \\
& =\Psi_{\max \left(t_{1}, t_{2}\right)}(f) .
\end{array}
$$


In addition, a granulometry is called Euclidean if

$$
\begin{aligned}
\Psi_{t}(T(f)) & =T\left(\Psi_{t}(f)\right), \\
\Psi_{t}(f) & =S_{t}\left(\Psi_{1}\left(S_{1 / t}(f)\right)\right),
\end{aligned}
$$

where $T$ is a translation and $S_{t}$ is a scaling of $t^{14}$.

The pattern spectrum of a granulometry is given by:

$$
P S_{t}(f)(x)=\Psi_{t}(f)(x)-\Psi_{t+\Delta t}(f)(x),
$$

where $\Delta t$ is a sampling parameter of the scale, which traditionally has been set to one.

The opening function, $O F$, is defined for binary functions as:

$$
O F(f)(x)=\max \left\{t \mid x \in \Psi_{t}(f)\right\} .
$$

Alternatively, this function can be computed at every point by taking the scale at which the pattern spectrum attains its maximum:

$$
O F(f)(x)=\arg \max _{t}\left\{P S_{t}(f)(x)\right\}
$$

The latter formulation has the advantage that it can be applied both to binary and gray-scale functions. The pattern spectrum can also be normalized by $f(x)$ in order to get a probability density function ${ }^{15}$.

The most used granulometry is generated by means of the opening operation of mathematical morphology with convex structuring elements (SEs). The opening with respect to a $\mathrm{SE}, b$, is given by:

$$
f \circ b=(f \ominus b) \oplus b,
$$

where $\ominus$ and $\oplus$ are the erosion and dilation morphological operations respectively. In gray-scale, these operations are given by:

$$
\begin{aligned}
& (f \ominus b)(x)=\min _{y}[f(x+y)-b(y)], \\
& (f \oplus b)(x)=\max _{y}[f(x+y)+b(y)] .
\end{aligned}
$$

A relevant property is that these granulometries are Euclidean if $f$ and $b$ are continuous functions.

\section{THICKNESS ESTIMATION IN GRAY-SCALE THROUGH GRANULOMETRIES}

Granulometries with ball-shaped SEs are of special interest for thickness estimation. As already mentioned, twice the opening function computed with openings with ball-shaped SEs of different sizes is equivalent to compute local thickness through HR for binary images ${ }^{12}$. Thus, the use of gray-scale mathematical morphology can be used to estimate thickness in gray-scale with some modifications. This can better be seen through an example.

Figure 2 shows a 1D signal and the pattern spectrum at two different locations. This is an ideal rectangular signal of thickness 4 with a simulated partial volume effect generated by a shift of $1 / 3$ of a voxel. Intuitively, the process of computing thickness in 1D can be divided into three steps. First, different boxes of different sizes $2 t$ are moved along the signal in such a way that every tested box is completely enclosed by and is as close as possible to the signal (see the dotted curves on Fig. 2). The resulting curves can be seen as approximations of the original signal at different scales. Thanks to the anti-extensivity property, the gray-scale tends to be reduced as the scale of the boxes is increased, as seen on the figure. Second, the pattern spectrum can be computed as the reduction in the gray-scale between two subsequent scales through (8). For example, the pattern spectrum at $2 t=4$ corresponds to the difference between the red and blue dotted curves on Fig. 2. Finally, local thickness is computed through (10). Instead of the boxes used in $1 \mathrm{D}$, a set of disks and a set of balls of radius $t$ are used in $2 \mathrm{D}$ and $3 \mathrm{D}$ respectively.

The same example can be used to show two problems of applying the aforementioned methodology and our proposed solution. The first problem is that, unlike the binary case, the pattern spectrum in gray-scale can have 

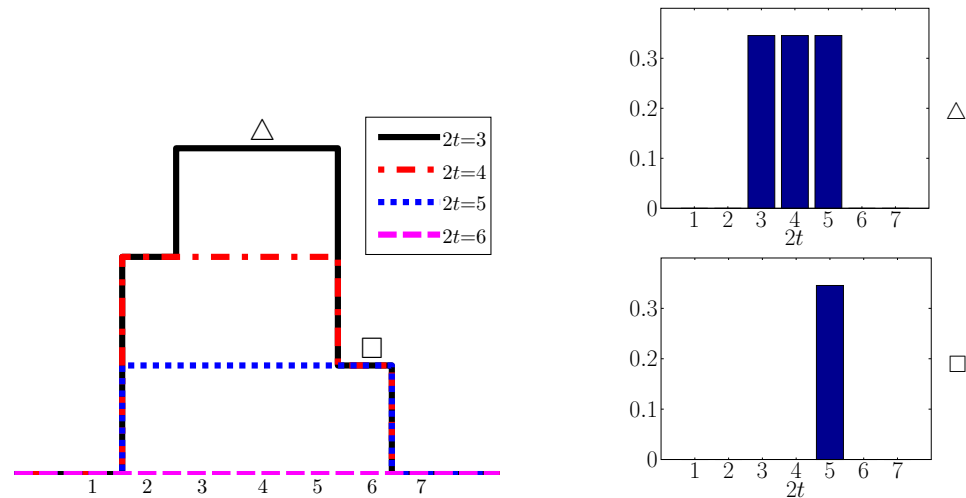

Figure 2. Left: opening of a 1D signal (in black) with balls of different sizes. Right: pattern spectrum at the two marked locations in the signal (triangle and square). The signal simulates the partial volume effect from a shift of $1 / 3$ of a voxel.

more than one peak. Figure 2 shows that the pattern spectrum at a specific location can attain the maximum value at different scales (see the triangle). Consequently, it seems more appropriate to compute local thickness as a combination of the values at the pattern spectrum rather than only considering the peak as in (10). The second problem is that computing thickness through the opening function can lead to overestimations at edges, since the values yielded by (10) do not depend on the local gray-scale. For example, the thickness estimated using (10) at the square on Fig. 2 is 5 . Assuming that the gray-scale values are generated by partial volume effects, the result obtained through granulometry must be adjusted, considering that only a part of the voxel at an edge belongs to the object. The correction can be performed by subtracting from the initial estimation the percentage of voxel that does not belong to the object. Thus, the following proposed formula takes into account the two aforementioned issues:

$$
T h(f)(x)=2 \frac{\sum t P S_{t}(f)(x)}{\sum P S_{t}(f)(x)}-\frac{M-f(x)}{M-m},
$$

with $M$ and $m$ being the maximum and minimum values of $f$ respectively. The first term uses the pattern spectrum as a weighting function of the radius $t$ and the second term corrects bad estimations at edges generated by partial volume effects. Notice that, since variations on the gray-scale can also be generated by noise, it is necessary to preprocess the image in order to reduce the level of noise.

Furthermore, the pattern spectrum must be computed in the range of the expected thickness. In particular, considering too large values of $t$ is inconvenient, since it is expensive, and the pattern spectrum of remaining noisy voxels will have peaks at large values of $t$, generating biases in the thickness estimation. This effect can be avoided by stopping the estimation of the pattern spectrum when the morphological opening reduces the range of values of the image to a certain percentage of the original range, which can be adjusted depending on the remaining noise level. Moreover, considering too small values of $t$ is also inconvenient, since the estimation of thickness will be more exposed to discretization errors, as those described in ${ }^{6}$.

A final aspect to consider is the integration of local estimations of thickness into a global measurement. Considering that the estimations of local thickness at interior voxels are usually more accurate than at borders, the gray-scale values can also be used as a weighting function for computing global thickness as:

$$
T h(f)=\frac{\sum f(x) T h(f)(x)}{\sum f(x)} .
$$

The estimation of global thickness for the example on Fig. 2 by averaging the results of (10) is 3.6, 4.2 or 4.8, and it is $3.33,4.08$ or 4.83 by using (10) and (15), where the different values are obtained depending on which maximum is taken for the voxels at the marked triangle. In turn, the estimated thickness through (14) and (15) is 4.08 and does not have any dependency. 


\section{DISCRETE IMPLEMENTATIONS OF GRANULOMETRIES}

There are two additional implementation issues to consider when continuous granulometries are applied to discrete data. In practice, morphological openings with ball-shaped SEs generate a granulometry only for continuous functions $f$ and $b$. Unfortunately, working implementations require discretizations that can lead to inaccuracies ${ }^{16}$. Thus, discretization is an important factor that has to be considered carefully. It is worth mentioning that the problems described in this section also affect the accuracy of the HR method. The following subsections describe the proposed solutions to the issues generated by the discretization of continuous granulometries.

\subsection{Discretization of SEs}

The first implementation issue is the discretization of a ball-shaped SEs. Let $b_{t, c}$ be the discretization of the continuous ball $B_{t, c}$. Discrete flat SEs (i.e., SEs where $b_{t, c}(x)=0$ if $x \in N$, and $-\infty$ otherwise, for a neighborhood $N$ ) have the advantage that the output of the openings only depend on the values of the image ${ }^{11}$. Thus, the effect of the SE in the opening is reduced to define a neighborhood in which the minimum and maximum operations in (12) and (13) are applied. However, this type of SE is inconvenient for granulometric analysis, since the approximation of ball-shaped SEs through flat SEs is poor. As a consequence, implementations with flat SEs can lead to families of functions that do not generate granulometries ${ }^{17}$.

An alternative to deal with this problem is the use of discrete non-flat SEs, in which the SE can take values different from 0 and $-\infty$. The design of this SE must consider that it must be as flat as possible in order to reduce the dependency of the computations on the values of the SE, must be in agreement with the range of gray-scale values in the image, and must ensure that the output remains in the original range of gray-scale values. The following family of discrete non-flat SEs complies with these restrictions:

$$
b_{t, c}(x)= \begin{cases}(M-m) \cdot\left[\operatorname{Vol}\left(v(x) \cap B_{t, c}\right)-1\right], & \text { if } v(x) \cap B_{t, c} \neq \emptyset \\ -\infty, & \text { otherwise }\end{cases}
$$

where $v$ is the voxel centered at $x$, and $\mathrm{Vol}$ stands for the volume. The volume of every voxel is assumed to be equal to one. The proposed SE has some interesting properties. First, it is almost flat, except for a thin annular region along the boundary of the ball where values vary from 0 to $-(M-m)$. In consequence, most of the advantageous properties of flat SEs are preserved. Second, the radius $t$ can take fractional values, and not only integer values. Third, $b_{t, c}$ can be easily translated to points $c$ different from the origin. This facilitates the estimations of thickness with subvoxel accuracy. Finally, $b_{t, c}$ is related to the volume of the ball. Indeed:

$$
\int_{v(x) \cap B_{t, c} \neq \emptyset} \operatorname{Vol}\left[v(x) \cap B_{t, c}\right] d x=\operatorname{Vol}\left(B_{t, c}\right)=\frac{4}{3} \pi t^{3},
$$

That means that an erosion (dilation) computes the minimum (maximum) value in a determined volume, where values of $b_{t, c}$ different from 0 and $-\infty$ are used to deal with partial volume effects.

\subsection{Translation Invariance}

The second implementation issue is related to translation invariance. Although granulometries with ball-shaped SEs are translation-invariant for continuous functions, they are not for discrete implementations ${ }^{15}$. That means that, in general:

$$
O F(T(f)) \neq T(O F(f))
$$

for a translation $T$. Nevertheless, the error induced by the non-translation invariance in the estimation of thickness is limited. Thus, with some considerations, the estimation of thickness can be obtained with a small error.

In order to show this point, an experiment with rectangular 1D signals of different sizes was conducted whose results can also be extrapolated to higher dimensions. Instead of translating the signal and leaving the SE static, the SE was translated around in the range of one unit, provided that the results of both procedures are equivalent for testing the translation invariance of the estimations. Table 1 shows the range of the estimated thickness for 
Table 1. Range of estimated thickness with subpixel accuracy (r) and maximum error (e) for 1D rectangular signals of different sizes and different $\Delta t$

\begin{tabular}{c|cc|cc|cc|cc|ccc}
\hline$\Delta t$ & \multicolumn{1}{|c|}{} & \multicolumn{1}{c}{ Size of the Rectangle } & \multicolumn{3}{|c}{} \\
& $\mathrm{r}$ & $\mathrm{e}$ & $\mathrm{r}$ & $\mathrm{e}$ & $\mathrm{r}$ & $\mathrm{e}$ & $\mathrm{r}$ & $\mathrm{e}$ & $\mathrm{r}$ & $\mathrm{e}$ \\
\hline 0.5 & $2.0-4.0$ & 2.0 & $2.0-5.0$ & 3.0 & $2.0-6.0$ & 4.0 & $2.0-7.0$ & 5.0 & $2.0-8.0$ & 6.0 \\
1.0 & $2.0-4.5$ & 2.0 & $2.0-5.5$ & 3.0 & $2.0-6.5$ & 4.0 & $2.0-7.5$ & 5.0 & $2.0-8.5$ & 6.0 \\
1.5 & $2.5-4.0$ & 1.5 & $3.5-5.0$ & 1.5 & $4.5-6.0$ & 1.5 & $5.5-7.0$ & 1.5 & $6.5-8.0$ & 1.5 \\
2.0 & $2.5-4.0$ & 1.5 & $3.5-5.0$ & 1.5 & $4.5-6.0$ & 1.5 & $5.5-7.0$ & 1.5 & $6.5-8.0$ & 1.5 \\
2.5 & $2.5-4.0$ & 1.5 & $3.5-5.0$ & 1.5 & $4.5-6.0$ & 1.5 & $5.5-7.0$ & 1.5 & $6.5-8.0$ & 1.5 \\
3.0 & $2.0-4.0$ & 2.0 & $3.0-5.0$ & 2.0 & $4.0-6.0$ & 2.0 & $5.0-7.0$ & 2.0 & $6.0-8.0$ & 2.0 \\
\hline
\end{tabular}

Table 2. Thickness estimation (Th) for blurred bars of different thicknesses using HR for three thresholds $(\alpha)$ and TG

\begin{tabular}{|c|c|c|c|c|c|c|c|c|}
\hline \multirow{3}{*}{ Thickness } & \multicolumn{6}{|c|}{ HR } & \multirow{2}{*}{\multicolumn{2}{|c|}{ TG }} \\
\hline & \multicolumn{2}{|c|}{$\alpha=0$} & \multicolumn{2}{|c|}{$\alpha=0.5$} & \multicolumn{2}{|c|}{$\alpha=0.9$} & & \\
\hline & Th & error & $\mathrm{Th}$ & error & $\mathrm{Th}$ & error & Th & error \\
\hline 5 & 10.93 & $118.6 \%$ & 4.98 & $0.4 \%$ & 2.99 & $40.2 \%$ & 4.61 & $7.8 \%$ \\
\hline 10 & 15.88 & $58.8 \%$ & 9.95 & $0.5 \%$ & 7.98 & $20.2 \%$ & 9.89 & $1.1 \%$ \\
\hline 20 & 25.71 & $28.6 \%$ & 19.82 & $0.9 \%$ & 17.85 & $10.8 \%$ & 19.67 & $1.7 \%$ \\
\hline
\end{tabular}

different 1D signals with known thickness for $c$ of $b_{t, c}$ varying from -0.5 to 0.5 with a step of 0.05 . In addition, the table shows the results for different values of $\Delta t$, which is the parameter in (8).

As seen on the table, small values of $\Delta t$ are inconvenient, since the pattern spectrum can attain maxima at different values of $t$ and the estimations are more exposed to discretization errors. On the other hand, large values of $\Delta t$ are also inconvenient, since the range of estimated thickness is expanded, leading to a loss in accuracy. The best results are obtained for the range $1.5 \leq \Delta t \leq 2.5$. For these values, the estimation of thickness at one specific location is accurate with a maximum error of 1.5 units.

This means that, e.g., if $\Delta t=2$, the thickness estimation at one specific location inside the voxel (e.g., the center of the voxel) using a specific $c$ for $b_{t, c}$ (e.g., the origin) can be used as a representative thickness estimation for all the points inside the voxel with a maximum error of 1.5 units. The consequence of this finding is that, the computation of local thickness with subvoxel accuracy can be avoided in applications where an error of 1.5 units is affordable for $\Delta t=2$. Estimations of thickness with a smaller error can still be obtained by computing thickness in up-sampled versions of the image by a factor of two obtained through interpolation. This up-sampling has been applied in Sect. 5, since the resolution of the tested images was rather limited.

Finally, it is important to remark that current methods that make use of partial differential equation- (PDE) based mathematical morphology ${ }^{18,19}$ are inconvenient for granulometric analysis, since they usually shift the range of gray-scale values, which has a direct impact on the estimation of the pattern spectrum, and have problems dealing with critical points where sections of evolving curves are close to each other.

\section{RESULTS}

Experiments have been conducted on synthetic models and images acquired through micro computed tomography $(\mu \mathrm{CT})$ and cone beam computed tomography (CBCT).

A first experiment has been designed to compare HR with the proposed method, referred to as TG (thickness through granulometry). Thickness for blurred versions of 2D bars of different sizes have been computed. These bars have been blurred with a Gaussian filter with a standard deviation of one. Parameter $\Delta t$ has been set to 2. Table 2 compares the results of HR for three different thresholds $(\alpha)$ with the results yielded by TG through (15). In order to make a fair comparison, the proposed improvements to deal with discretization errors of Sect. 4 have been applied to both HR and TG. As can be seen, the results yielded by HR strongly depend on the threshold. In contrast, TG is able to yield values close to the ground-truth without binarization. Thus, TG is more appropriate for images where the segmentation process is not trivial. As aforementioned, both methods are equivalent for binary images. 


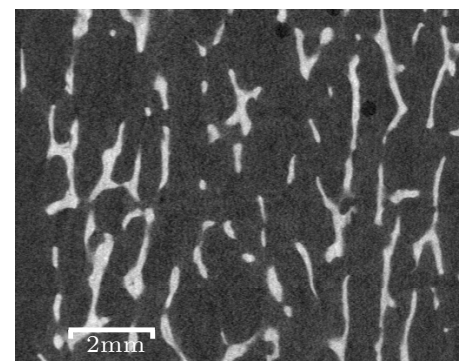

(a)

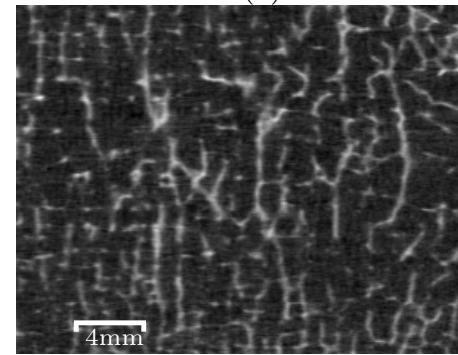

(b)

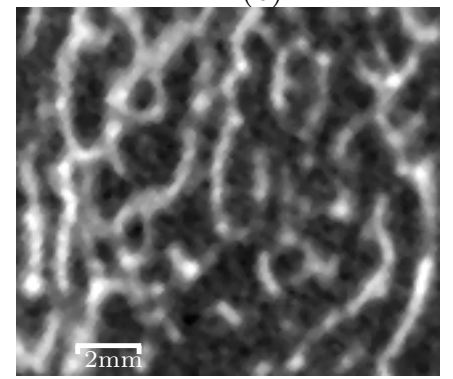

(c)

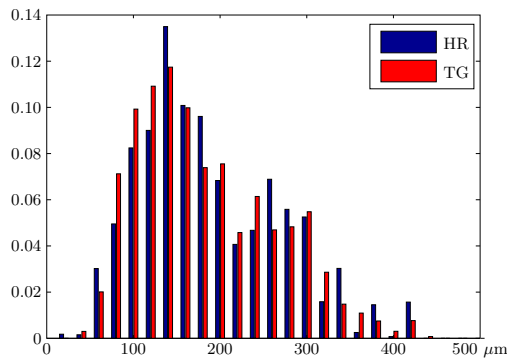

(d)

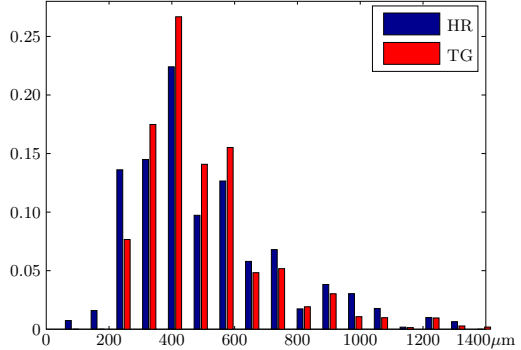

(e)

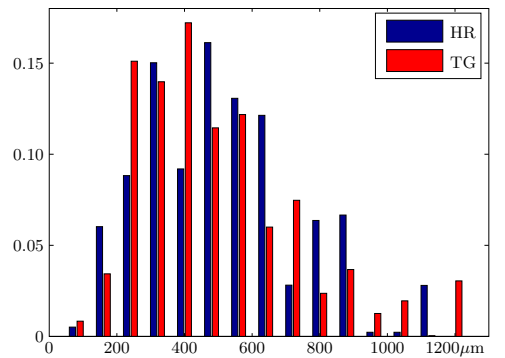

(f)

Figure 3. (a,b): Slices of a $\mu \mathrm{CT}$ scan of a radius and a vertebra respectively. (c) Slice of a CBCT scan of a radius. (d-f): Thickness distributions using HR and TG in 2D of the images (a-c) respectively.

Figure 3 shows 2D slices of a micro computed tomography $(\mu \mathrm{CT})$ scan of a radius and a vertebra, and a slice of a cone beam computed tomography $(\mathrm{CBCT})$ scan of a radius, and their corresponding thickness distributions computed in 2D through HR and TG. Figure 4 shows renderings of the same scans, and their corresponding thickness distributions computed in 3D through HR and TG. The images have an isotropic resolution of $20 \mu m$ and $82 \mu \mathrm{m}$ for the $\mu \mathrm{CT}$ images of the radius and vertebra respectively, and $80 \mu \mathrm{m}$ for the CBCT image of the radius. The images have been carefully segmented in order to compute HR. The processing for the tested images has been stopped when the range of values is reduced to $25 \%$ of the original range in order to avoid biases generated by noisy voxels (cf. Sect. 3).

Visual inspection shows that the thickness distributions computed through both methods are quite similar for all images. Table 3 shows the global thicknesses computed through (15) for both methods. As can be seen, HR and TG also yield similar values of global thickness with small absolute differences. The advantage of TG is that, without segmentation, it yields similar estimations of thickness to the ones obtained through HR on carefully segmented versions of the original images. Thus, TG is more convenient for images with limited resolution.

\section{DISCUSSION}

This paper has presented an extension of the HR method to estimate thickness in gray-scale. In addition to the use of gray-scale mathematical morphology, the extension deals with specific issues of the use of gray-scale and issues derived from the use of granulometries in discrete data in order to increase the accuracy of the estimations. Results show that the measurements in binary yielded by HR strongly depend on the binarization 


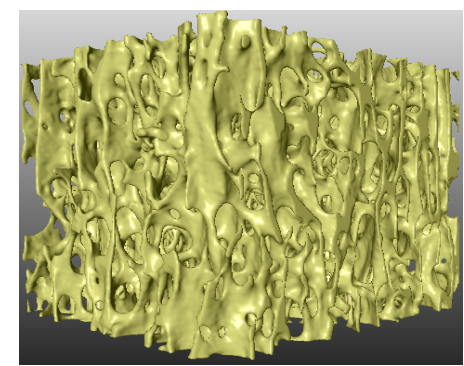

(a)

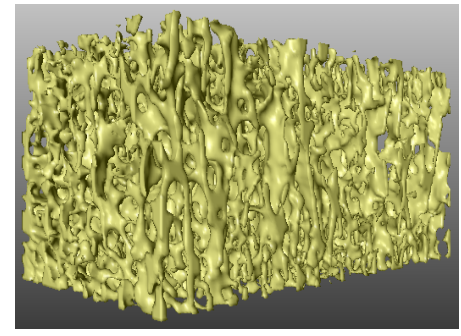

(b)

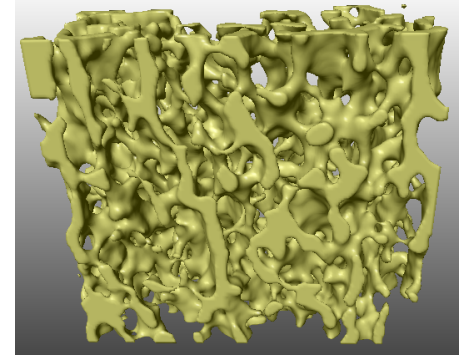

(c)

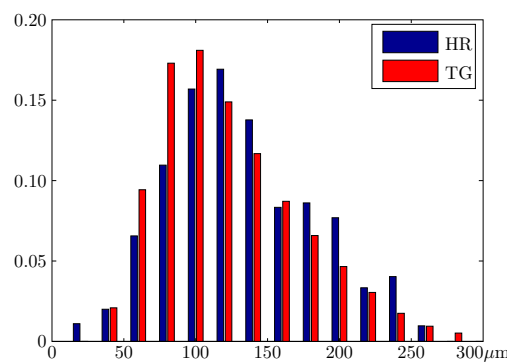

(d)

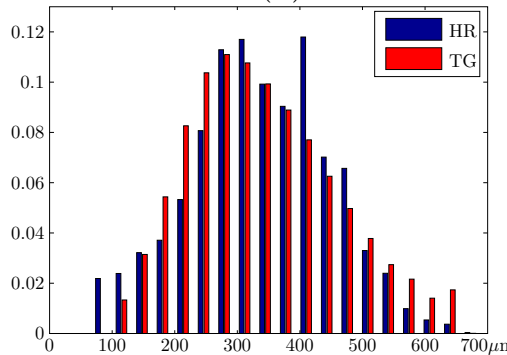

(e)

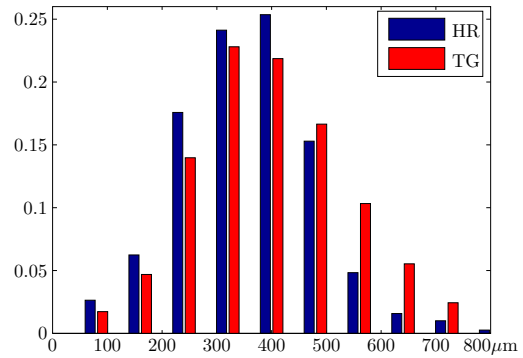

(f)

Figure 4. (a,b): Renderings of a $\mu \mathrm{CT}$ scan of a radius and a vertebra respectively. (c) Rendering of a CBCT scan of a radius. (d-f): Thickness distributions using HR and TG in 3D of the images (a-c) respectively.

Table 3. Global thickness computed for the images of Figs. 3 and 4.

\begin{tabular}{l|ccc|ccc}
\hline & \multicolumn{3}{|c|}{$2 \mathrm{D}$} & \multicolumn{3}{c}{$3 \mathrm{D}$} \\
Dataset & HR $(\mu \mathrm{m})$ & TG $(\mu \mathrm{m})$ & Abs. Diff. $(\%)$ & HR $(\mu \mathrm{m})$ & TG $(\mu \mathrm{m})$ & Abs. Diff. $(\%)$ \\
\hline$\mu$ CT radius & 195.80 & 197.73 & 0.99 & 142.41 & 140.68 & 1.21 \\
$\mu$ CT vertebra & 531.33 & 526.63 & 0.88 & 350.80 & 355.03 & 1.21 \\
CBCT radius & 535.55 & 510.81 & 4.62 & 400.82 & 397.67 & 0.79 \\
\hline
\end{tabular}

process, whereas the proposed extension does not, making the latter more appropriate for images acquired in vivo, where the segmentation process is not trivial.

Plans for future research include performing an extended validation of the proposed method including trabecular bone from more skeletal sites.

\section{ACKNOWLEDGEMENTS}

This research has been supported by the Swedish Research Council (VR), grant no. 2006-5670. We thank Prof. Osman Ratib from the Service of Nuclear Medicine of the Geneva University Hospitals for providing the $\mu \mathrm{CT}$ data of the vertebra, Andres Laib from SCANCO and Torkel Brismar from the Karolinska Institute for providing the $\mu \mathrm{CT}$ data of the radius, and Eva Klintström from the CMIV at Linköping University for providing the CBCT data of the radius. 


\section{REFERENCES}

[1] Pernow, Y., Hauge, E., Linder, K., Dahl, E., and Sääf, M., "Bone histomorphometry in male idiopathic osteoporosis," Calcif. Tissue Int. 84, 430-438 (2009).

[2] Kleerekoper, M., Villanueva, A., Stanciu, J., Rao, D., and Parfitt, A., "The role of three-dimensional trabecular microstructure in the pathogenesis of vertebral compression fractures," Calcif. Tissue Int. 37, 594-597 (1985).

[3] Hunter, D., Gerstenfeld, L., Bishop, G., Davis, A. D., Mason, Z., Einhorn, T., Maciewicz, R., Newham, P., Foster, M., Jackson, S., and Morgan, E., "Bone marrow lesions from osteoarthritis knees are characterized by sclerotic bone that is less well mineralized," Arthritis Res. $\mathcal{E}$ Ther. 11(1), R11 (2009).

[4] Thomsen, J. S., Ebbesen, E. N., and Mosekilde, L., "Predicting human vertebral bone strength by vertebral static histomorphometry," Bone 30(3), $502-508$ (2002).

[5] Mitton, D., Cendre, E., Roux, J.-P., Arlot, M., Peix, G., Rumelhart, C., Babot, D., and Meunier, P., "Mechanical properties of ewe vertebral cancellous bone compared with histomorphometry and high-resolution computed tomography parameters," Bone 22(6), 651-658 (1998).

[6] Hildebrand, T. and Rüegsegger, P., "A new method for the model-independent assessment of thickness in three-dimensional images," J. Microsc. 185(1), 67-75 (1997).

[7] Saha, P. K. and Wehrli, F. W., "Measurement of trabecular bone thickness in the limited resolution regime of in vivo MRI by fuzzy distance transform," IEEE Trans. Med. Imag. 23(1), 53-62 (2004).

[8] Saha, P. K., Wehrli, F. W., and Gomberg, B. R., "Fuzzy distance transform: Theory, algorithms, and applications," Comput. Vis. Image Underst. 86, 171-190 (2002).

[9] Darabi, A., Chandelier, F., and Baroud, G., "Thickness analysis and reconstruction of trabecular bone and bone substitute microstructure based on fuzzy distance map using both ridge and thinning skeletonization," Can. J. Elect. Comput. Eng. 34(1/2), 57-62 (2009).

[10] Krug, R., Carballido-Gamio, J., Burghardt, A. J., Haase, S., Sedat, J. W., Moss, W. C., and Majumdar, S., "Wavelet-based characterization of vertebral trabecular bone structure from magnetic resonance images at $3 \mathrm{~T}$ compared with micro-computed tomographic measurements," Magnetic Resonance Imaging 25(3), $392-398$ (2007).

[11] Matheron, G., [Random Sets and Integral Geometry], John Wiley \& Sons (1975).

[12] Coeurjolly, D., "Fast and accurate approximation of the Euclidean opening function in arbitrary dimension," Proc. Int. Conf. Pattern Recognit. (ICPR), 229-232 (2010).

[13] Soille, P., [Morphological Image Analysis, Principles and Applications], Springer, 2 ed. (2004).

[14] Dougherty, E. R., "Euclidean gray-scale granulometries: Representation and umbra inducement," J. Math. Imaging Vis. 1, 7-21 (1992).

[15] Luengo Hendriks, C. L., van Kempen, G. M. P., and van Vliet, L. J., "Improving the accuracy of isotropic granulometries," Pattern Recognit. Lett. 28(7), 865-872 (2007).

[16] Luengo Hendriks, C. L. and van Vliet, L. J., "Using line segments as structuring elements for samplinginvariant measurements," IEEE Trans. Pattern Anal. Mach. Intell. 27(11), 1826-1831 (2005).

[17] Vogt, R., "Morphological operator distributions based on monotonicity and the problem posed by digital disk-shaped structuring elements," in [SPIE Proc. Digit. and Opt. Shape Represent. and Pattern Recognit.], Juday, R., ed., 938, 1019-1022 (1988).

[18] Sapiro, G., Kimmel, R., Shaked, D., Kimia, B. B., and Bruckstein, A. M., "Implementing continuous-scale morphology via curve evolution," Pattern Recognit. 26(9), 1363-1372 (1993).

[19] Breuß M. and Weickert, J., "Highly accurate schemes for PDE-based morphology with general convex structuring elements," Int. J. Comput. Vis. 92(2), 132-145 (2011). 